\title{
DESCRIPCIÓN DE LOS ESTADOS INMADUROS DE RUTELA LINEOLA (LINNEO, 1767) (COLEOPTERA: MELOLONTHIDAE: RUTELINAE)
}

\author{
Viviana Calisto y Enrique Morelli ${ }^{1}$ \\ ${ }^{1}$ Sección Entomología, Facultad de Ciencias. Iguá 4225. Montevideo, URUGUAY. \\ E-mail: emorelli@fcien.edu.uy
}

Calisto, V. \& E. Morelli. 2011. Descripción de los estados inmaduros de Rutela lineola (Linneo, 1767). Acta Zool. Mex. (n. s.), 27(1): 67-76.

RESUMEN. En el presente trabajo se describen e ilustran la larva de tercer estadio y la pupa de Rutela lineola Linneo, 1767. Este rutelino es bastante común en el Uruguay, sus larvas son xilófagas encontrándoselas en troncos caídos. Se presenta una clave para las especies de Rutelinae descritas para el Uruguay incluyendo a $R$. formosa y $R$. dorcyi.

Palabras Clave: Morfología, estados inmaduros, taxonomía, rutelinos, Uruguay.

Calisto, V. \& E. Morelli. 2011. Description of the immature stages of Rutela lineola (Linnaeus, 1767) (Coleoptera: Melolonthidae: Rutelinae). Acta Zool. Mex. (n. s.), 27(1): 67-76.

ABSTRACT. In the present work are described and illustrated the third-instar larva and pupa of Rutela lineola Linneo, 1767. This rutelinae is quite common in Uruguay, their larvae are xylophagous finding them inside fallen trunks. An identification key of the third-instar larvae for the Rutelinae species described in Uruguay including $R$. formosa and $R$. dorcyi, is presented.

Key Words: Morphology, immature stages, taxonomy, ruteline, Uruguay.

\section{INTRODUCCIÓN}

El género Rutela está muy diversificado en la región Neotropical donde habitan 29 especies (Jameson 1997), de las cuales solo se han descrito la larva de R. formosa Burmeister, 1844 (Ritcher 1966) y la larva y la pupa de $R$. dorcyi (Oliver 1789) (Jameson 1997). Rutela lineola Linneo 1767, es un rutelino distribuido entre Colombia y Argentina, muy común en el Uruguay. Los imagos se alimentan de flores de diversas plantas especialmente rosales y existen registros que se alimentan de sarandíes, $\mathrm{Ce}$ phalanthus glabratus (Rubiaceae) (Bentancourt et al. 2009). Moneé (1970) cita para el Uruguay 17 especies de rutelinos, de los cuales se han descrito la larva de Homonyx chalcea Blanchard, 1850 (Morelli 1996), y la larva de Macraspis dichroa cribrata Waterh., 1881 (Monné 1969). Con la descripción de la larva de otra especie se podrán 
valorar los caracteres sistemáticos comparándolos con los ya existentes, aportando nuevos datos para realizar una diagnosis general de la subfamilia Rutelinae, así como seleccionar aquellos que puedan separar las larvas de Rutela de los otros Rutelini en el Nuevo Mundo.

\section{MATERIALES Y MÉTODOS}

Se recogieron 7 larvas maduras dentro de galerías de un tronco caído procedente de la ciudad de Tacuarembó (Departamento de Tacuarembó) en el mes de septiembre de 2007. Dos larvas se sacrificaron por inmersión en agua hirviendo y se fijaron 24 hs en fijador Pampel. Luego se conservaron en alcohol 70\%. El resto de las larvas se mantuvieron vivas para obtener pupas y adultos. Se fijó una pupa en alcohol 70\%. Los adultos y las larvas preservados están depositados en la colección de Entomología de la Facultad de Ciencias de Montevideo. Los caracteres sistemáticos larvarios y pupales fueron descritos de acuerdo a la terminología propuesta por Böving (1936) y Ritcher (1966). Para la descripción de la quetotaxia torácica y abdominal se utilizó el sistema de fórmulas ideado por Morón (1987).

\section{RESULTADOS}

\section{Descripción de la larva de $3^{\circ}$ estadio (Figs. 1-18)}

En reposo (Fig. 1), cuerpo arqueado, subcilíndrico. Coloración blanca lechosa, cabeza y patas castaño. Dorsa de los segmentos torácicos y abdominales con leve pubescencia. Longitud $x: 33 \mathrm{~mm}$.

Cápsula cefálica (Fig. 2) esclerotizada, redondeada. Ancho máximo: $5 \mathrm{~mm}$, largo máximo: $4,8 \mathrm{~mm}$. Tegumento levemente reticulado color castaño a castaño oscuro. Sutura epicraneal (ES) corta y sinuosa. Suturas frontales (FS) prolongadas hasta la base de las antenas, con 4 sedas dorso-epicraneales (DES). Frente (F) levemente punteada con 4 sedas posteriores (PFS) y 2 sedas anteriores (AFS). Cada ángulo frontal anterior con una única seda larga y fina (AA). Clípeo trapezoidal dividido transversalmente en postclípeo proximal (PSC) y preclípeo distal (PC). Antenas (Fig. 16) de 4 segmentos desprovistos de sedas, con 2 sedas finas en la base (ASB). Los tres primeros segmentos subiguales. Tercero con un proceso apical interno (PAI). Último segmento corto y piriforme con hasta 3 máculas sensoriales ventrales y 4 dorsales grandes, ovaladas y claramente definidas (DSS). Labro (Fig. 3) largo y estrecho, tegumento levemente punteado. Borde clipeal casi recto, con 24 sedas anteriores (ALLS y ACLS) y 9 sedas posteriores (PLLS). Zona media con 2 sedas largas y finas (PCLS) emergiendo de crestas transversales internas, y 15 sedas internas cortas y finas (ILbS). Epifaringe (Fig. 4) ovalada. Dexiotorma (DX) transversal y alargado. Laeotorma (LT) casi transverso, con pternotorma (PTT) alargado. Nesia (NE) pre- 

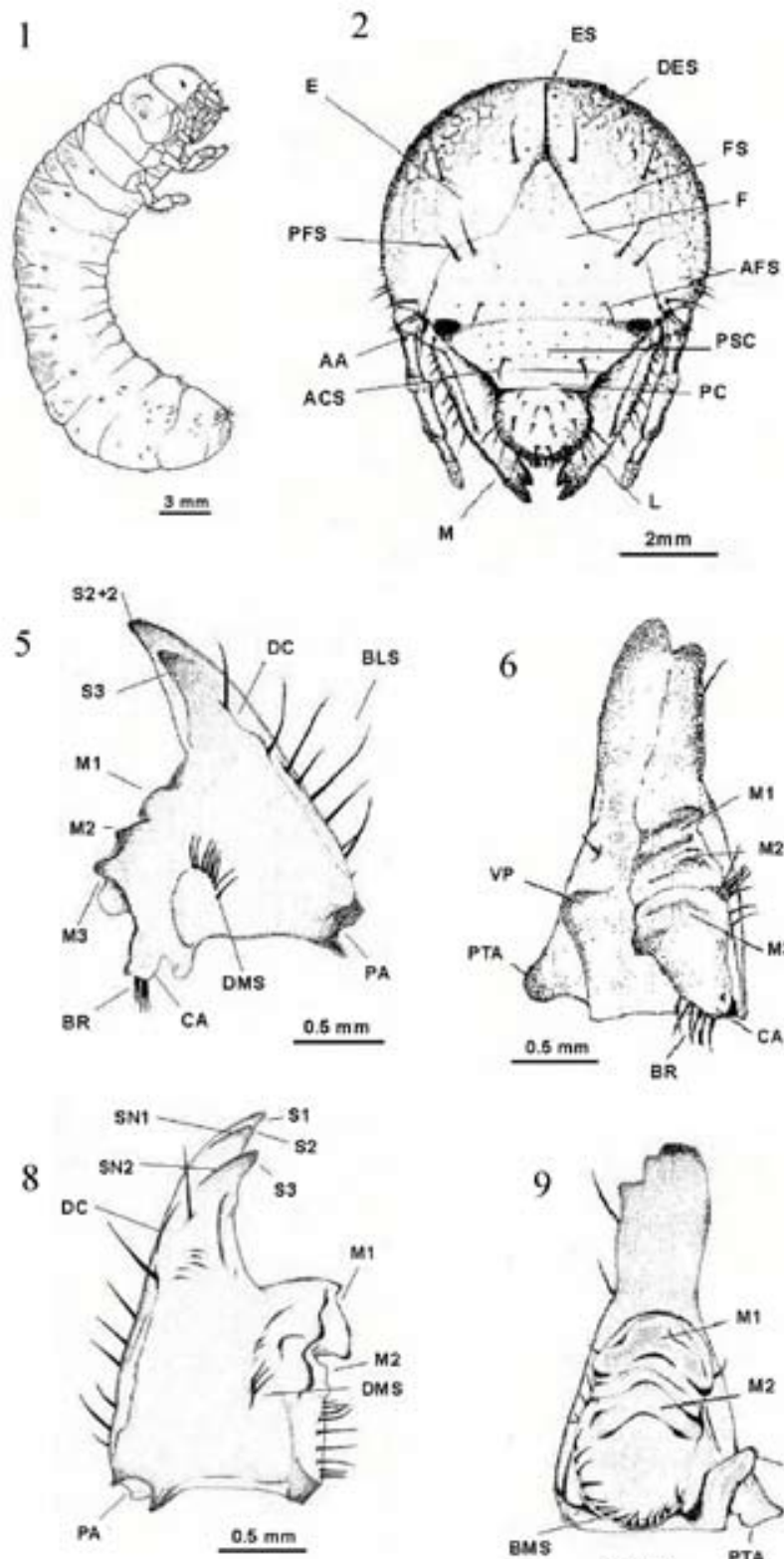
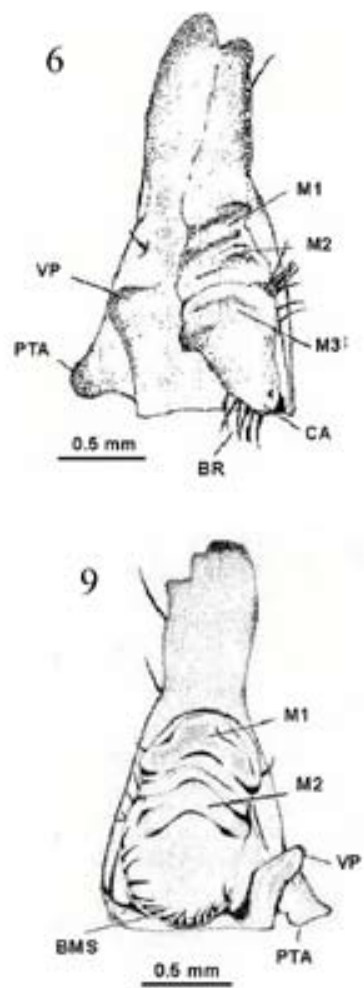
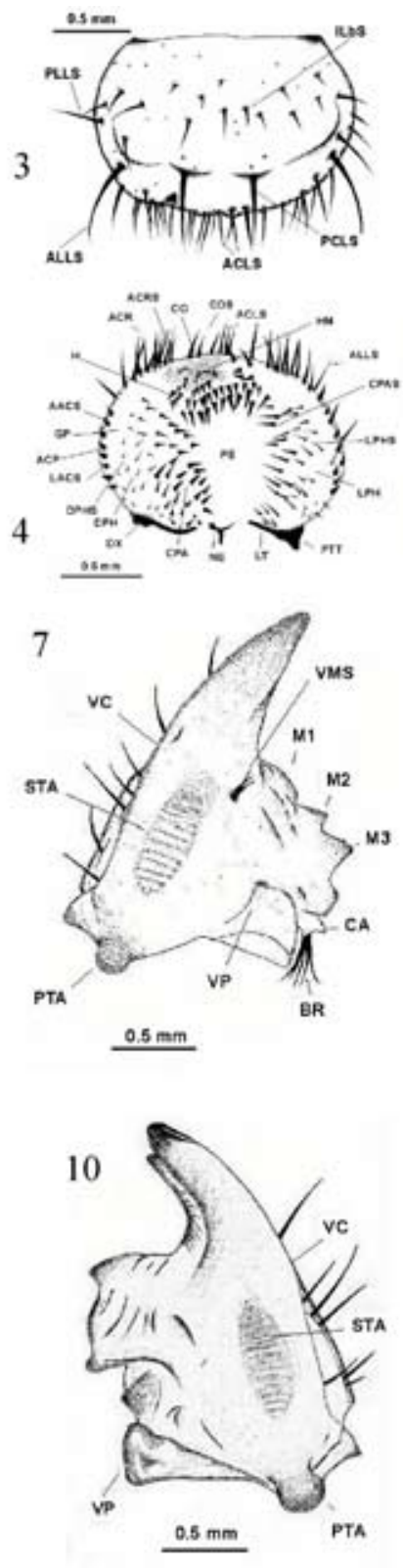

Figuras 1-10. Larva de tercer estadio de Rutela lineola. 1) vista lateral. 2) Cápsula cefálica, vista frontal. 3) Labro. 4) Epifaringe. 5) Mandíbula derecha, vista dorsal. 6) Mandíbula derecha, vista mesial. 7) Mandíbula derecha, vista ventral. 8) Mandíbula izquierda, vista dorsal. 9) Mandíbula izquierda, vista mesial. . 10) Mandíbula izquierda, vista ventral. 
sente con forma de "Y". Acroparia (ACR) y corypha (CO) unidos, con 23 sedas largas y finas (ACRS y COS). Haptómero (HM) presente con 37 heli (H). Chaetoparia (CPA) bien desarrollada, con más de 40 sedas (CPAS) cada vez más gruesas hacia el pedium (PE). Acantoparia (ACP) con 17 sedas cortas, gruesas, curvadas hacia delante (AACS y LACS). Dexiophoba (DPH) y Laeophoba (LPH) con numerosas sedas finas (DPHS y LPHS). Epizigum, proplegmata y plegmatia ausentes. Mandíbulas asimétricas, subtriangulares con base ensanchada. Coloración castaño oscuro a negro. Mandíbula derecha (Figs. 5, 6 y 7) con 3 dentículos incisivos $\left(S_{1+2}\right)$ y $S_{3}$. $S_{3}$ muy visible y separado de los anteriores por una escotadura ( $\mathrm{SN}$ ). Región molar amplia con 3 lóbulos molares. $\mathrm{M}_{1}$ y $\mathrm{M}_{2}$ pequeños y algo ensanchados. $\mathrm{M}_{3}$ cuadrangular y más voluminoso con brustia (BR) formada por 6 sedas largas y finas. Dorsalmente se destaca una carena (DC) con 2 sedas largas y finas, sedas alargadas próximas al borde externo (BLS) y 9 sedas cortas y finas (DMS) en hilera curva próximas a la región molar. Vista ventral con importante área estridulatoria (STA) ovalada y claramente definida con más de 14 hileras granulares, distalmente 3 sedas emergen de un gran poro (VMS). Mandíbula izquierda (Figs. 8, 9 y 10) con una importante área incisiva con 3 dentículos bien definidos separados por dos comisuras $\left(\mathrm{SN}_{1}\right.$ y $\left.\mathrm{SN}_{2}\right) . \mathrm{SN}_{2}$ se prolonga como un surco bordeado por 2 sedas largas y finas. Area molar formada por 2 dentículos. $\mathrm{M}_{1}$ voluminoso y ensanchado, $\mathrm{M}_{2}$ pequeño, triangular y limitado en su base por 15 sedas cortas y finas (BMS). Vista dorsal con 3 sedas cortas y finas (DMS) agrupadas. Borde externo con 6 sedas largas y finas (BLS). Vista ventral con área estridulatoria (STA) ovalada y algo ensanchada, con más de 16 hileras granulares bien definidas. Ambas mandíbulas con área estridulatoria distal menos definida, y con un proceso esclerotizado ventral (VP) entre el cóndilo de articulación ventral (PTA) y el área molar. Maxila (Figs. 11 y 12) con cardo (CAR) cuadrangular con numerosas sedas internas cortas y finas (ICARS). Mala (MA) presente con la línea de unión entre galea y lacinia poco visible. Uncus de la lacinia (LU) con base ancha y punta roma, rodeado por 3 sedas gruesas (LUS). Dorsal y ventralmente la lacinia (LA) presenta abundantes sedas largas y finas (IDLAS, EDLAS e IVLAS). Galea (GA) con sedas gruesas (IDGS) en región apical. Estipe (ST) con hilera de 9 dentículos estridulatorios (SD) cónicos (Fig. 13), uno en posición más alejado. Se destacan 2 procesos estridulatorios anteriores (ASDP), uno más pequeño y menos voluminoso. La hilera de dentículos está bordeada por numerosas sedas cortas y finas (PSTS y ASTS) y una seda lateral larga y fina (LSTS). Palpo maxilar (MP) con 4 artejos ensanchados, $1^{\circ} \mathrm{y}$ $3^{\circ}$ subiguales, $4^{\circ}$ segmento ovalado. Tercer artejo con 2 sedas ventrales largas y finas (VMPS). Palpífero (PF) con una seda dorsal corta y fina (SPF). Labio-hipofaringe (Fig. 14) con 2 lóbulos laterales (LL), ambos con sedas finas. Lóbulo izquierdo con un pequeño proceso lateral (PL). Entre ambos lóbulos se destaca un pequeño proceso hipofaringeal (TP) y un escleroma hipofaríngeo (HSC) voluminoso con un vértice hacia el centro. Lóbulo central de las glosas (GL) con 12 sedas cortas y gruesas 

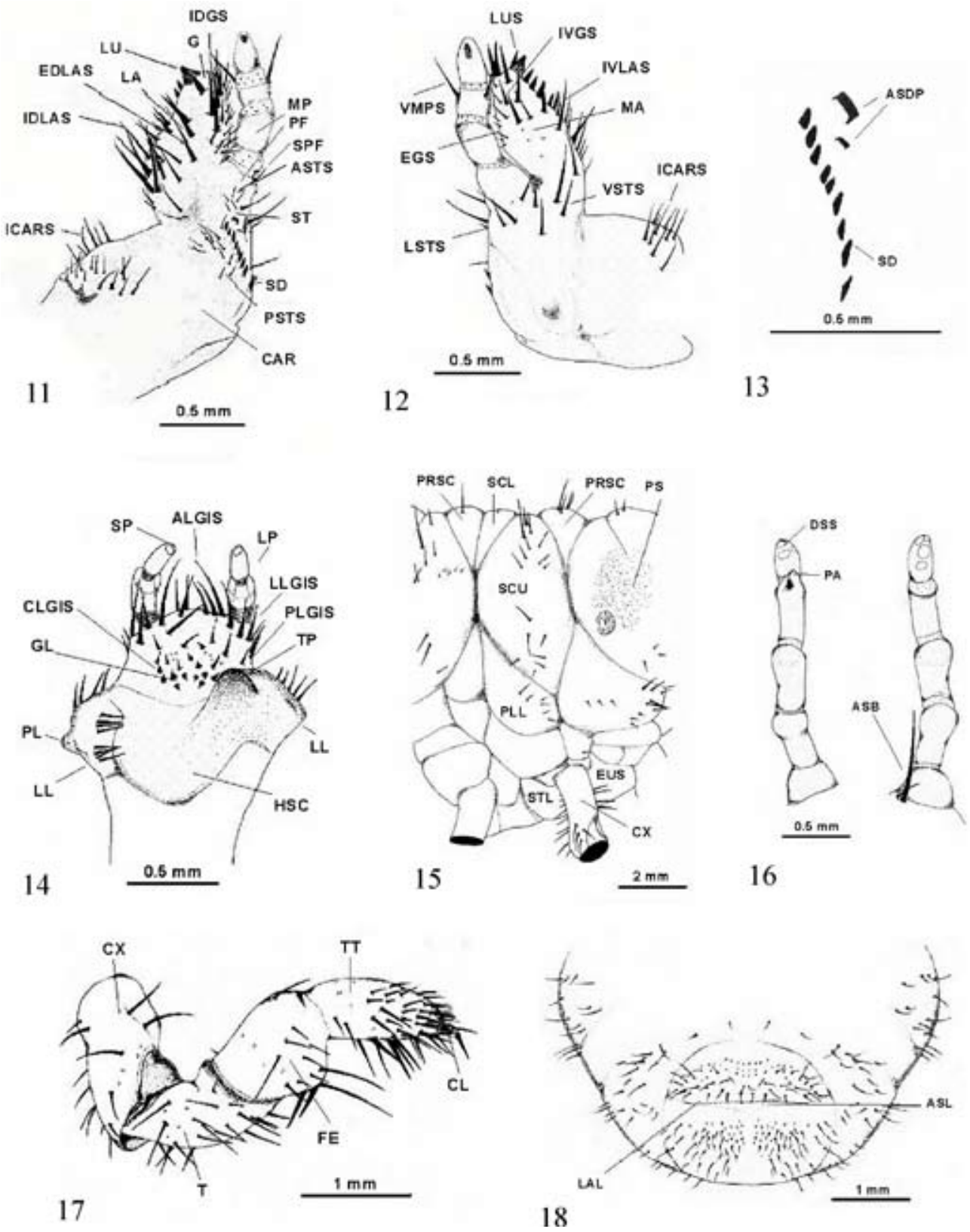

Figuras 11-18. Larva de tercer estadio de Rutela lineola. 11) Maxila, vista dorsal. 12) Maxila, vista ventral. 13) Dientes estridulatorios de la maxila. 14) Labio.15) Mesotórax, vista lateral. 16) Antena derecha vista ventral y dorsal. 17) Pata protorácica. 18) Raster. 
(CLGIS). Lóbulos laterales con más de 10 sedas finas (LLG1S y PLGIS) y de 6-10 sedas anteriores largas y finas (AGIS). Palpos labiales (LP) con 2 artejos, el $2^{\circ}$ más estrecho y corto terminado en una placa sensorial (SP) grande y ovalada.

Tórax (Fig. 15). Protórax con una placa supraespiracular (PS) poco regular de color castaño amarillento. Presenta un par de estigmas respiratorios con la concavidad dispuesta caudalmente. Mesotórax con preescudo (PRSC) con LF 1(2); Escudo (SCU) con LF 1(7), CF 1(5); Escutelo (SCL) con LF 1(1). Patas (Fig. 1) ligeramente curvadas hacia delante. Pata II y III subiguales. Pata I (Fig. 17) pequeña. Cada pata con una coxa (CX) larga y cilíndrica. Trocánter (T) largo, fémur corto y ensanchado con 2 sedas largas y gruesas, y numerosas sedas largas y finas. Tibio-tarso (TT) fusionado, muy pubescente y terminado en una uña (CL) con 2 sedas.

Abdomen (Fig. 1) con 8 estigmas respiratorios con la concavidad dirigida caudalmente. La quetotaxia de los segmentos abdominales no es constante. Dorso del segmento I: CE 1(10), LF 1(3) intercaladas; CE irregular (67), LF 1(5) intercaladas; CE irregular (54). Dorso del segmento II: CE 1(29), LF 1(1) intercaladas; CE irregular (106), LF 1(6) intercaladas; CE irregular (66). Dorsa de los segmentos III al VII: CE irregular (34), LF 1(1) intercalada; CE irregular (97), LF 1(8) intercaladas; CE irregular (66). Dorso del segmento VIII: CF 1(4), LF 1(2) intercaladas; CF 1(5), LF 1(2) intercaladas. Dorso del segmento X: LF 1 (16), CF 1(11) intercaladas. Raster (Fig. 18) sin palidia. Con la siguiente quetotaxia: CE (66-68), CF (18-20) y LF (8084). Área anal ventral con una franja transversal de sedas pequeñas y espiniformes intercaladas con sedas largas y finas dirigidas hacia el labio anal. Área anal dorsal con sedas espiniformes repartidas en dos sendas bien definidas separadas por un espacio central intercaladas con sedas finas. Hendidura anal (ASL) ligeramente curva.

\section{Descripción de la pupa (Figs. 19-21)}

Pupa exarata, tegumento amarillento, glabro sin ornamentaciones. Ancho máximo del cuerpo: $12 \mathrm{~mm}$, largo: $22 \mathrm{~mm}$. Pronoto ensanchado. Mesonoto triangular con base estrecha. Abdomen con 6 pares de espiráculos en los segmentos dorsales. Cuatro pares de gin-traps (Hinton, 1946) ubicados en el centro tergal de los segmentos $2 / 3$, $3 / 4,4 / 5,5 / 6$. Pterotecas alargadas, con bordes redondeados. La primera pteroteca llega hasta el $2^{\circ}$ segmento abdominal mientras que la segunda pteroteca se prolonga hasta el $3^{\circ}$. Los últimos segmentos abdominales son más pequeños. Margen externo del último segmento presenta numerosas sedas cortas.

Clave para larvas de las especies de Rutelinae descritas para el Uruguay incluyendo R. formosa y R. dorcyi

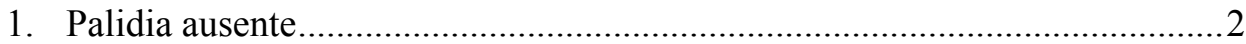

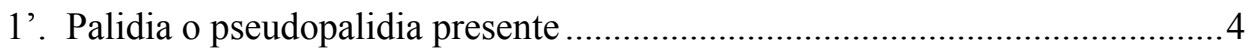




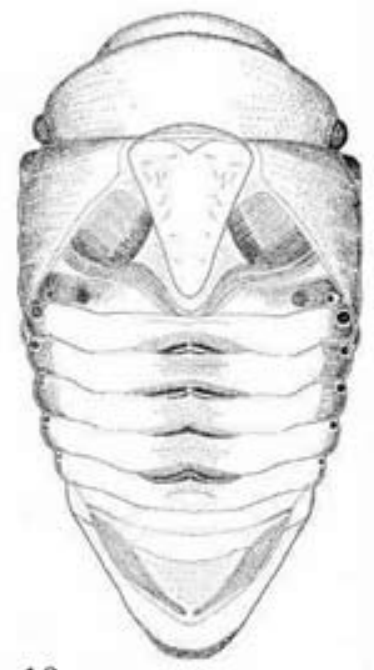

19

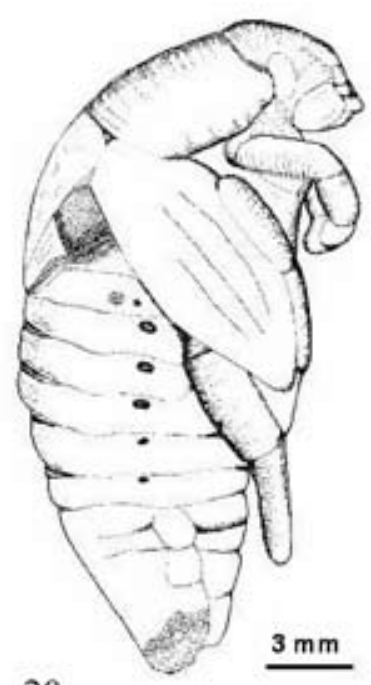

20

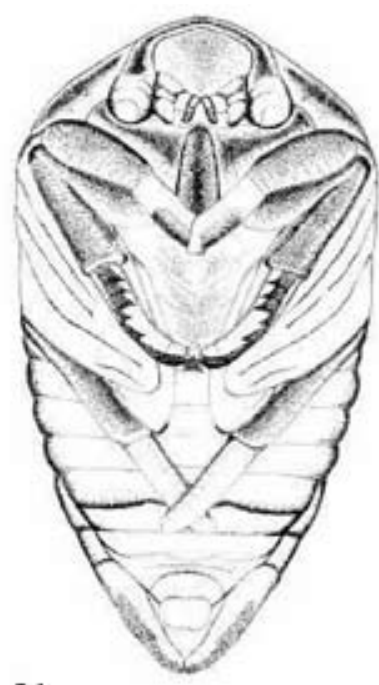

21

Figuras 19-21. Pupa de Rutela lineola. 19) vista dorsal. 20) vista lateral. 21) vista ventral.

2. Con 4 sedas dorsoepicraneales. Maxilas con 9 dientes estridulatorios y dos procesos truncados anteriores Rutela lineola

2'. Con 2-5 sedas dorsoepicraneales. Maxilas con 8-10 dientes estridulatorios y un proceso truncado anterior.

3. Con 2 sedas dorsoepicraneales. Maxilas con 8-10 dientes estridulatorios y un proceso truncado anterior Rutela formosa

3'. Con 5 sedas dorsoepicraneales. Maxilas con 8-9 dientes estridulatorios y un proceso truncado anterior Rutela dorcyi

4. Palidia presente. Maxilas con 7 dientes estridulatorios ..... Macraspis dichroa

4'. Pseudopalidia presente con 4 hileras de sedas cortas y espiniformes. Maxila con 9 dientes estridulatorios y un proceso truncado anterior Homonyx chalcea

\section{DISCUSIÓN}

La larva de tercer estadio y la pupa de Rutela lineola comparten los caracteres anatómicos propios de los rutelinos. La presencia de un nesium con epitormae posterior conspicuo en la epifaringe, es un carácter suficiente para identificar la larva de Rutela lineola. La quetotaxia del raster serviría para diferenciar primariamente las 3 especies de Rutelinae que han sido descritos para el Uruguay. El número de sedas dorsoepicraneales y de procesos truncados maxilares completaría la diagnosis de las especies $R$. dorcyi y $R$. formosa, dentro de la clave general presentada. La pupa de Rutela 
lineola puede llegar a diferenciarse de la de $R$. dorcyi por la proporción ancho-largo del pronoto, el número de espiráculos abdominales visibles y la forma general del mesonoto. Jameson (1997) no menciona la presencia de " gin traps" centrotergales en la descripción de esta última.

\section{LITERATURA CITADA}

Bentancourt, C., I. Scatoni \& E. Morelli. 2009. Insectos del Uruguay. Agencia Nacional de Innovación e Investigación; Comisión Sectorial de Investigación Científica. Universidad de la República. $658 \mathrm{pp}$.

Böving, A. 1936. Description of the larvae of Plectis aliena Chapin and explanation of new terms applied to the epipharynx and raster. Proceedings of the Entomological Society of Washington, 38: 169-185.

Hinton, H. 1946. The "gin traps" of some beetle pupae: aprotective device which appears to be unknown. Transactions of the Royal Entomological Society of London, 97: 473-496.

Jameson, M. L. 1997. Phylogenetic analysis of the subtribe Rutelina and revision of the Rutela generic groups (Coleoptera: Scarabaeidae: Rutelinae: Rutelini). Bulletin of the University of Nebraska, 14: 1-184.

Monné, M. 1969. Descripción del último estadio larval de Macraspis dichroa cribata Waterh., Blaesia atra Burm. y Marmarina tigrina (Gory \& Perch.) (Coleoptera, Scarabaeidae). Revista Brasileira de Biologia, 29: 367-376.

Monné, M. 1970. Fauna de los coleópteros del Uruguay. Fac. Agronomía, Montevideo- Uruguay.

Morelli, E. 1996. Descripción de la larva y de la pupa de Homonyx chalcea Blanchard, 1950 (Coleoptera, Scarabaeidae, Rutelinae). Acta Zoológica Mexicana (n. s.), 68: 53-60.

Morón, M. A. 1987. Los estados inmaduros de Dynastes hyllus Chevrolat (Coleoptera, Melolonthidae, Dynastinae y Rutelinae). Anales del Instituto de Biología, UNAM, Serie Zoología, 47: 119-134.

Ritcher, P. O. 1966. White grubs and their allies. A study of North American Scarabaeoid larvae. Studies in Entomology No. 4. Oregon State University Press, Corvallis.

\footnotetext{
Abreviaturas utilizadas en las figuras:

AA- seda cefálica del ángulo anterior

AACS- seda anterior del acantoparia

ACLS- seda anterior del lóbulo central del labro

ACP- acantoparia

ACR- acroparia

ACRS- seda del acroparia

ACS- seda clipeal anterior

AFS- seda frontal anterior

ALGIS- seda del lóbulo anterior de la glosa

ALLS- seda anterior del lóbulo lateral del labro

ASB- seda basal antenal

ASDP- proceso estridulatorio anterior

ASL- hendidura anal

ASTS- seda anterior del estipe

BLS- seda borde lateral
}

ANEXO 
BMS- seda basal molar

BR- brustia

CA- calus

CAR- cardo

CL- uña

CLGIS- seda lóbulo central de la glosa

CO- corypha

COS- seda del corypha

CPA- chaetoparia

CPAS- seda del chaetoparia

CX- coxa

DC- carena dorsal

DES- seda dorso-epicraneana

DMS- seda dorsal mandíbular

DPH- dexiophoba

DPHS- sedas del dexiophoba

DSS- máculas sensoriales

DX-dexiotorma

E- epicráneo

EDLAS- seda dorsal externa de la lacinia

EGS- seda externa de la galea

ES- sutura epicraneal

EUS- euesterno

F- frente

FE- fémur

FS- sutura frontal

G- galea

GL- glosa

GP- gimnoparia

H- heli

HM- haptómero

HSC- escleroma hipofaringeal

ICARS- seda interna del cardo

IDGS- seda dorsal interna de la galea

IDLAS- seda dorsal interna de la lacinia

ILbS- seda labral interna

IVGS- seda ventral interna de la galea

IVLAS- seda ventral interna de la lacinia

L- labro

LA- lacinia

LACS- seda lateral del acroparia

LAL- labio anal inferior

LL- lóbulo lateral

LLGIS- seda del lóbulo lateral de la glosa

LP- palpo labial

LPH- laeophoba

LPHS- seda del laeophoba 
LSTS- seda lateral del estipe

LT- laeotorma

LU- uncus de la lacinia

LUS- sedas uncus

M- mandíbula

M1,2,3- dientes molares

MA- mala

MP- palpo maxilar

NE- nesia

PA- preartis

PAI- proceso apical interno

PC- preclípeo

PCLS- seda posterior del lóbulo central del labro

PE- pedium

PF- palpífero

PFS- seda frontal posterior

PL- proceso lateral

PLGIS- seda latero-posterior de la glosa

PLL- lóbulo pleural

PLLS- seda lateral posterior del labro

PRSC- preescudo

PS- placa supraespiracular

PSC- postclípeo

PSTS- seda posterior del estipe

PTA- postartis

PTT- pternotorma

PS- placa sensorial

$S$ 1,2,3- dientes incisivos

SCL- escutelo

SCU- escudo

SD- dientes estridulatorios

SN 1,2- escotaduras

SPF- seda palpífero

ST- estipe

STA- área estridulatoria

STL- esternelo

$\mathrm{T}$ - trocánter

TP- proceso hipofaringeal

TT- tibio-tarso

VC- carena ventral

VMPS- seda ventral del palpo maxilar

VMS- seda ventral mandibular

VP- proceso ventral

VSTS- seda ventral del estipe 